\title{
The optical system for JASMINE and the CCD centroiding experiment
}

\author{
Taihei Yano ${ }^{1}$, Naoteru Gouda ${ }^{1}$, Yukiyasu Kobayashi ${ }^{1}$, \\ Takuji Tsujimoto ${ }^{1}$, Tadashi Nakajima ${ }^{1}$, Hideo Hanada ${ }^{1}$, \\ Yoshiyuki Yamada ${ }^{2}$, Hiroshi Araki ${ }^{3}$, Seiichi Tazawa ${ }^{3}$, \\ Kazuyoshi Asari ${ }^{3}$, Seiitsu Tsuruta ${ }^{3}$, Nobuyuki Kawano ${ }^{3}$ \\ and Naruhisa Takato ${ }^{4}$ \\ ${ }^{1}$ National Astronomical Observatory of Japan, Mitaka, Tokyo 181-8588, Japan \\ email: yano.t@nao.ac.jp, naoteru.gouda@nao.ac.jp, yuki@merope.mtk.nao.ac.jp, \\ taku.tsujimoto@nao.ac.jp, tadashi@dodgers.mtk.nao.ac.jp, hanada@miz.nao.ac.jp \\ ${ }^{2}$ Graduate School of Science, Kyoto University, Sakyo-ku, Kyoto 606-8502, Japan \\ email: yamada@scphys.kyoto-u.ac.jp \\ ${ }^{3}$ National Astronomical Observatory of Japan, Mizusawa, Iwate 023-0861, Japan \\ email: arakih@miz.nao.ac.jp, tazawa@miz.nao.ac.jp, asa@miz.nao.ac.jp,tsuruta@miz.nao.ac.jp, \\ kawano@miz.nao.ac.jp \\ ${ }^{4}$ Subaru Telescope, National Astronomical Observatory of Japan, 650 Noth A'ohoku Place, \\ Hilo, HI 96720, USA Hawaii \\ email: takato@subaru.naoj.org
}

\begin{abstract}
We have investigated the optical design for the Japan astrometry satellite mission (JASMINE). In order to accomplish measurements of astrometric parameters with high accuracy, optics with a long focal length and a wide focal plane for astrometry are required. In 1977 Korsch proposed a three mirror system with a long focal length and a wide focal plane. The Korsch system is one of the convincing models. However, the center of the field is totally vignetted because of the fold mirror. Therefore we consider an improved Korsch system in which the center of the field is not vignetted. Finally, we obtain the diffraction limited optical design with small distortion. Our project needs a common astrometric technique to obtain precise positions of star images on solid state detectors to accomplish its objectives. In order to determine the centers of stars, an image of the point source must be focused onto the CCD array with a spread of a few pixels. The distribution of photons (photoelectrons) over a set of pixels enables us to estimate positions of stars with sub-pixel accuracy. We modify the algorithm to estimate the real positions of stars from the photon weighted mean, which was originally developed by the FAME (Full-Sky Astrometric Mapping Explorer) group. Finally, we obtain the results from the experiment that the accuracy of estimation of distance between two stars has a variance of about $1 / 300$ pixel; that is, the error for one measurement is about $1 / 300$ pixel, which is almost an ideal result given by Poisson photon noise. We also investigate the accuracy of estimation of positions with a different size of PSF. In this case also, we find that the accuracy of estimation has a variance of about $1 / 300$ pixel.
\end{abstract}

\section{Optics for JASMINE}

We have investigated the optical design for the Japan astrometry satellite mission (JASMINE). JASMINE will measure parallaxes, positions and proper motions of stars in our Galaxy with a precision of $10 \mu \mathrm{arcsec}$ in order to study the fundamental structure and evolution of the disk and the bulge components of the Milky Way Galaxy. In order to accomplish such measurements with high accuracy, optics with a long focal length and 


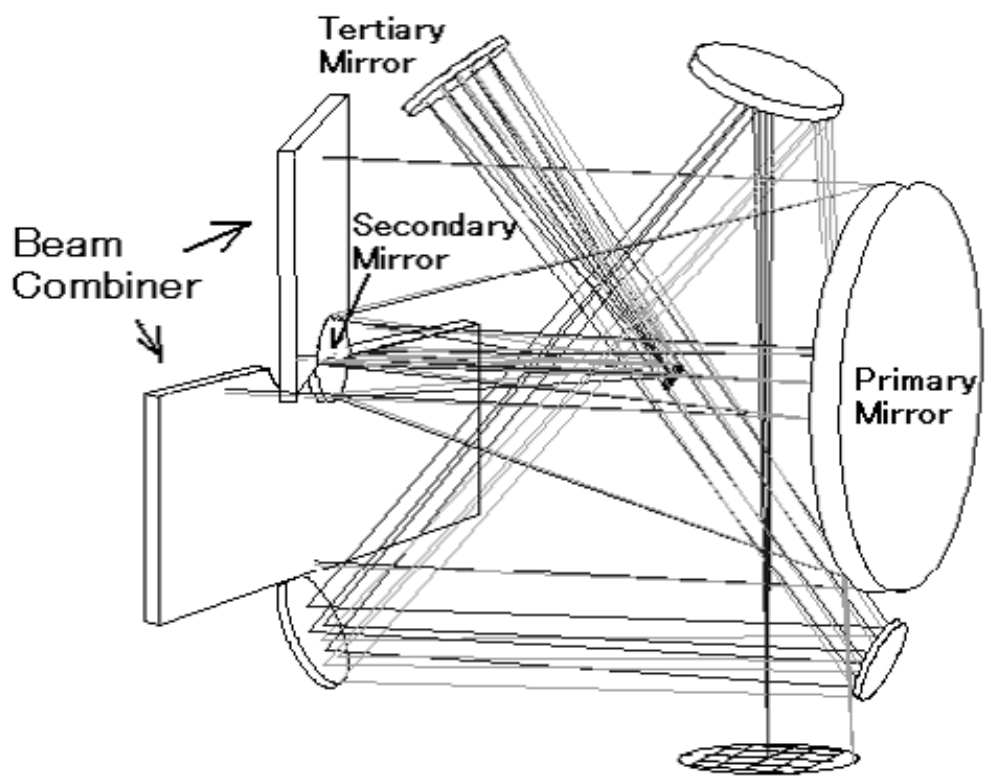

Focal Plane

Figure 1. A schematic of the JASMINE optics

a wide focal plane are required. Numerous modern telescope objectives are of RitchyChretien form, e.g., the Hubble space telescope, Subaru, and so on. A Ritchy-Chretien system has two mirrors, both of which are hyperboloids. This system is corrected for spherical aberration and coma, leaving astigmatism and strong field curvature uncorrected. This strong field curvature is hard to correct in such an optics. In 1977 Korsch proposed a three mirror system with a long focal length and a wide focal plane. The Korsch system is one of the convincing models. However, the center of the field is totally vignetted because of the fold mirror. Therefore we consider the improved Korsch system in which the center of the field is not vignetted. A schematic of the optics is shown in Fig. 1.

The aperture size of the optics is $1.5 \mathrm{~m}$, and its focal length, $f$, is $50 \mathrm{~m}$ in order to accomplish $f \lambda / D w=2$, where $\lambda, \mathrm{D}$, and $w$ is the wavelength, the aperture size, and pixel size, respectively. The size of the detector for $\mathrm{z}$-band is $6 \mathrm{~cm} \times 3 \mathrm{~cm}$ with $4096 \times 2048$ pixels. The pixel size is $15 \mu \mathrm{m}$ which corresponds to 61.9 milliarcsec (mas) on sky. These

Table 1. Summary of the instrument parameters

\begin{tabular}{cc}
\hline Optics design & Korsch System (3 mirrors) \\
\hline Aperture size & $1.5 \mathrm{~m}$ \\
Focal length & $50 \mathrm{~m}$ \\
pixel size & $15 \mu \mathrm{m}$ \\
pixel on sky & $61.9 \mathrm{mas}$ \\
Array size & $6 \mathrm{~cm} \times 3 \mathrm{~cm}$ \\
Pixels per detector & $4096 \times 2048$ \\
Number of detectors & $98(7 \times 14)$ \\
Basic Angle & 99.5 \\
\hline
\end{tabular}


Table 2. Surface data summary

\begin{tabular}{ccccccc}
\hline Surf & Type & Radius & Thickness & Glass & Diameter & Conic \\
\hline OBJ & STANDARD & Infinity & Infinity & & 0 & 0 \\
1 & STANDARD & Infinity & 2325 & & 1530.435 & 0 \\
STO & STANDARD & -5400 & -2175 & MIRROR & 1500.682 & -0.9861102 \\
3 & STANDARD & -1750.010 & 1312.5 & MIRROR & 322.9818 & -5.174046 \\
4 & COORDBRK & - & 0 & & - & - \\
5 & STANDARD & Infinity & 0 & MIRROR & 107.043 & 0 \\
6 & COORDBRK & - & -1350 & & - & - \\
7 & STANDARD & 2378.875 & 1350 & MIRROR & 450.1022 & -0.7149477 \\
8 & STANDARD & Infinity & 1275 & & 302.0378 & 0 \\
9 & COORDBRK & - & 0 & & - & - \\
10 & STANDARD & Infinity & 0 & MIRROR & 318.6309 & 0 \\
11 & COORDBRK & - & -2175 & & - & - \\
12 & COORDBRK & - & 0 & & - & - \\
13 & STANDARD & Infinity & 0 & MIRROR & 438.1686 & 0 \\
14 & COORDBRK & - & 2725 & & - & - \\
15 & COORDBRK & - & 0 & & - & - \\
16 & STANDARD & Infinity & 0 & MIRROR & 568.9629 & 0 \\
17 & COORDBRK & - & -2475 & & - & - \\
IMA & STANDARD & -14002.99 & & & 654.6421 & 0 \\
\hline
\end{tabular}

parameters are summarized in Table 1 . We also show the summary of the surface data in JASMINE optics in Table 2.

The image quality of the field of view has been analyzed. The spot diagram is shown in Fig. 2. This result shows that the field of view with diffraction limited image is achieved. Furthermore, the field distortion is also investigated. The value of distortion is $0.02 \%$ at maximum. This is enough small for our astrometry mission.

Finally we obtain the diffraction limited optical design with small distortion.

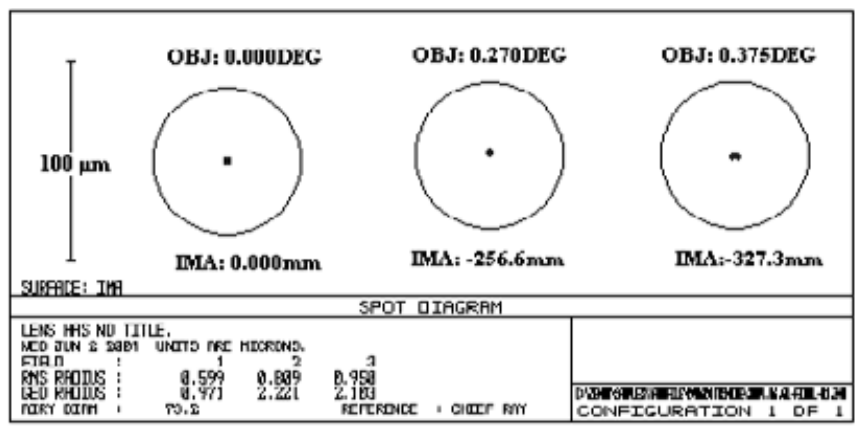

Figure 2. A spot diagram showing that a diffraction limited image is achieved.

\section{CCD Centroiding Experiment}

Measuring the centroiding of stars is one of the most important problems for astrometry. We examined the accuracy of the centroid of stars and obtained the relative distance of stars to an accuracy of 1/300 pixel (Yano et al. 2004); that is almost ideal. Our experimental method is shown below. 


\subsection{Algorithm}

In order to estimate the precise distance of two point sources in image frames to subpixel accuracy, the following algorithm is proposed. Here, we show the algorithm used in this experiment. Before the analysis, each image frame is bias subtracted and flat fielded. First, we pick two stars to measure the distance. Next we seek the pixel in which a number of photons is maximum in each star. Then we pick up a square subset of $5 \times 5$ pixels around the peak pixel of each star image. Accordingly, the number of photons is the maximum value at the center of pixels in both two stars. Only the pixel values of the two subsets are used to measure the distance of the two stars. We calculate the photon weighted mean of each star by the following equation.

$$
\left(\begin{array}{l}
x_{c} \\
y_{c}
\end{array}\right)=\frac{1}{\sum_{i} \sum_{j} N_{i j}}\left(\begin{array}{c}
\sum_{i} \sum_{j} N_{i j} i \\
\sum_{i} \sum_{j} N_{i j} j
\end{array}\right),
$$

where $N_{i j}$ is the number of photons at the position $(i, j)$. The photon weighted means $\left(x_{c}, y_{c}\right)$ derived by the above equation are different from the real positions $\left(x_{a}, y_{a}\right)$. Here, we assume that the difference between the photon weighted mean and the real position is proportional to the deviation of the photon weighted mean from the center of the pixel.

$$
x_{a}-x_{c}=k x_{c},
$$

where $k$ is a coefficient for the correction of the position of a star. This assumption was originally adopted by the FAME group for their laboratory experiment (Triebes et al. 1999, 2000). However, our treatment of the coefficient $k$ is different from that of the FAME group. Triebes et. al. regarded $k$ as a single parameter across the image frame, but we allow $k$ to be specific to each star. This way, we take into account the variation of the shape of PSF. We calculate the parameters, $k$, by using the least squares method. Then we obtain the real position $x_{a}$ from the estimated parameter, $k$. The algorithm, used in this experiment, is very useful. The reason is as follows. First, it is easy to calculate the photon weighted mean from the data. Second, we need not assume the shape of the PSF. We note that the shape of the PSF is assumed from the estimated parameter, $k$, implicitly.

Below we show the above algorithm explicitly. We define the positions of star1 and star2 as $x_{a 1}$ and $x_{a 2}$, respectively.

$$
\begin{aligned}
& x_{a 1}=x_{c 1}+k_{1} x_{c 1} \\
& x_{a 2}=x_{c 2}+k_{2} x_{c 2}
\end{aligned}
$$

where, $x_{c}$ is the photon weighting mean of a star.

Here we define a function $I$ as

$$
\begin{aligned}
& I=0\left(x_{c 2}>x_{c 1}\right) \\
& I=1\left(x_{c 2}<x_{c 1}\right) .
\end{aligned}
$$

The relative distance of the two stars $\left|\delta x_{a}\right|$ is

$$
\begin{aligned}
\left|\delta x_{a}\right| & =x_{a 2}-x_{a 1}+I \\
& =x_{c 2}-x_{c 1}+k_{2} x_{c 2}-k_{1} x_{c 1}+I \\
& =\left(1+k_{2}\right)\left(x_{c 2}-x_{c 1}\right)+\left(k_{2}-k_{1}\right) x_{c 1}+I \\
& \equiv \alpha \Delta+\beta x_{c 1}+I
\end{aligned}
$$

where, $\alpha=\left(1+k_{2}\right), \beta=\left(k_{2}-k_{1}\right)$, and $\Delta=\left(x_{c 2}-x_{c 1}\right)$. 

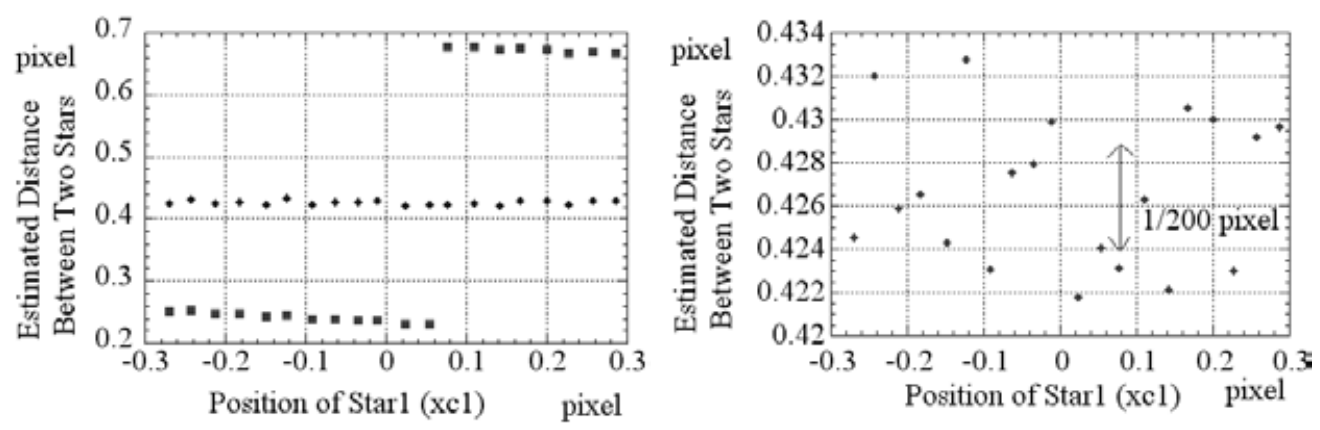

Figure 3. (Left panel) Relative distance between two stars against the photon weighted mean of star1. The squares indicate only the distances between photon weighted means of two stars, that is, no correction is performed. On the other hand, the diamonds indicate the estimated distances by linear correction of the photon weighted mean described here. In this experiment, $f \lambda / D w$ is equal to about 3. (Right panel) Same with the diamonds in the left panel.

We wish to derive the values of the parameters $k_{1}$ and $k_{2}$, with which the above equation is satisfied with the smallest error. In other words, we use a least squares method. So, we define $S$ as

$$
S=\sum\left(\alpha \Delta+\beta x_{c 1}+\gamma+I\right)^{2},
$$

where $\gamma=-\left|\delta x_{a}\right|$. The derivative of $S$ by each parameter is equal to zero. Then the following relations are satisfied.

$$
\begin{aligned}
& \frac{\partial S}{\partial \alpha}=\alpha \sum \Delta^{2}+\beta \sum \Delta x_{c 1}+\gamma \sum \Delta+\sum \Delta I=0, \\
& \frac{\partial S}{\partial \beta}=\alpha \sum \Delta x_{c 1}+\beta \sum x_{c 1}^{2}+\gamma \sum x_{c 1}+\sum x_{c 1} I=0, \\
& \frac{\partial S}{\partial \gamma}=\alpha \sum \Delta+\beta \sum x_{c 1}+\gamma \sum 1+\sum I=0 .
\end{aligned}
$$

From the above relations, we obtain the positions of two stars, $x_{a 1}$ and $x_{a 2}$ from the estimated parameters. Finally, we note again that the above least squares method is easy to calculate without explicit assumption of the PSF. Furthermore, it is an advantage for calculating thousands of stars because of its simplicity.

\subsection{Experimental results}

We have taken twenty image frames by sliding the CCD array. The interval of sliding is $1 \mu \mathrm{m}$; that is, twenty steps correspond to 1 pixel. From these twenty image frames, we estimate the distance of two stars, using the algorithm shown in the previous section. An image of the point spread function (PSF) of a star is focused onto the CCD array with a spread of about three pixels, using a lens with focal length of $200 \mathrm{~mm}$. The distance between the image field and the lens is about $570 \mathrm{~mm}$, and the length between the lens and the CCD camera is about $310 \mathrm{~mm}$. For a light source of the simulated stars, white light is used. The results for our experiment are shown in Fig. 3. The abscissa indicates the photon-weighted mean of star1. The ordinate indicates the distance between two stars.

Here, we note that the integer part of the distance is eliminated. Accordingly, the distance has a value between 0 and 1 . The squares show only the separations between photon-weighted means of two stars, that is, no correction is performed. On the other hand, the diamonds are the estimated distances between two stars by using the algorithm. 

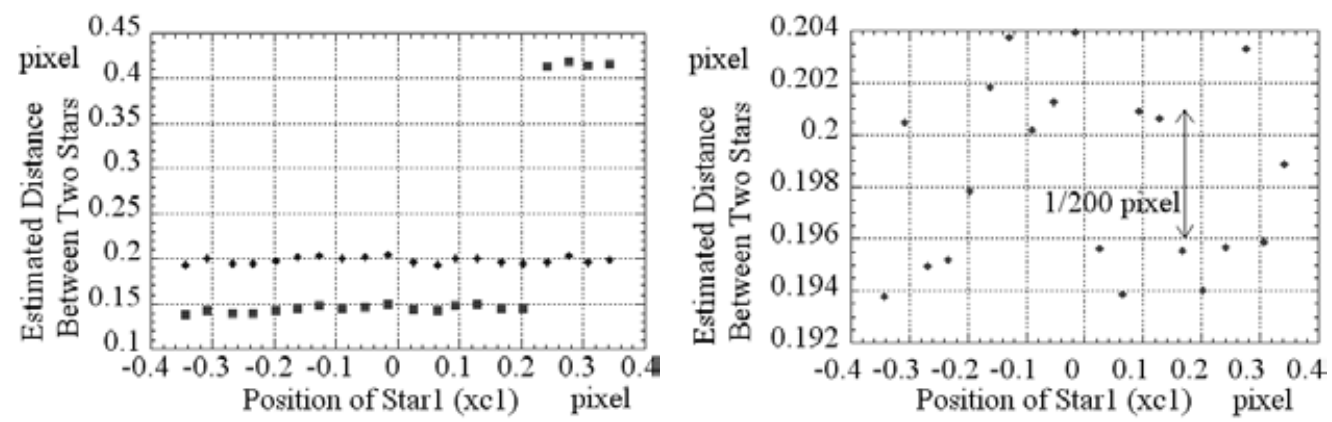

Figure 4. Same as Fig. 3, but $f \lambda / D w=1$.

Distances of two stars with no correction, that is, difference of photon-weighted means of two stars, are distributed to two groups, one is a value around 0.24 , and the other, around 0.68 . On the other hand, estimated distances using the linear correction, all the measurements are the values of around 0.43 . Then the accuracy of estimation becomes exceedingly high. These estimated distances (the diamonds) are shown again in the right panel of Fig. 3. As we can see, the variance of the estimated distances of two stars is about $1 / 300$ pixel; that is, the error of the estimation is $1 / 300$ pixel for one measurement, which is almost the ideal one given by the Poisson noise of photons.

Next, we investigate accuracy of estimation with a different size of PSF, using a lens with focal length of $100 \mathrm{~mm}$. The distance between the image field and the lens is about $730 \mathrm{~mm}$, and the length between the lens and the CCD camera is about $110 \mathrm{~mm}$. In this case, the image size of the PSF is about 1 pixel. The results are shown in Fig. 4 . The squares show only the separations between photon-weighted means of two stars. On the other hand, the diamonds are the estimated distances between two stars, which are shown again in the right panel of Fig. 4. In this case also, the accuracy of estimation is about $1 / 300$ pixel.

Here we consider the reason that the photon weighted mean differences between star1 and star2 are discontinuous. We pick up a square subset around the peak pixel of star image in this experiment. When the peak pixel moves to the adjoining pixel by sliding the CCD array, the region of a square subset changes. Accordingly, the calculated photon weighted mean jumps discontinuously. The analytical value of this discontinuity is $\frac{k_{2}}{k_{2}+1}$. This analytical form shows that the small discontinuity represents the small $k_{2}$. The value of the discontinuity in the first case of experiments is about $0.68-0.23 \simeq 0.45$. In this experiment, the estimated parameters $\alpha, \beta, \gamma, k_{1}$, and $k_{2}$ are $1.815,0.112,-0.427$, 0.703 , and 0.815 , respectively. The error in the experiment is about $3.3 \times 10^{-3}$. The value of $k_{2}$ is consistent with the discontinuity of 0.45 . In the second case, the discontinuity in Fig. 5 is about $0.41-0.14 \simeq 0.27$. In this case, these parameters, $\alpha, \beta, \gamma, k_{1}$, and $k_{2}$ are $1.363,-1.80 \times 10^{-2},-0.198,0.381$, and 0.363 , respectively. This discontinuity consistent with the value of $k_{2}$. Comparing these two cases, the discontinuity in Fig. 3 is larger than that in Fig. 4. This is because the value of $k_{2}$ in the first case is larger than that in the second case.

For comparison, we estimate the distance of the two stars with the algorithm in which we use a common parameter $k$ for the two stars, that is, $k_{1}=k_{2}$ is satisfied. This algorithm is essentially the same with that in FAME. In this case we find that the accuracy of estimation is about 1/100 pixel, which is worse than the above results obtained from our algorithm. 


\section{Concluding remarks}

The three mirror optical design for the Japan astrometry satellite mission has been studied. In order to accomplish measurements of astrometric parameters with high accuracy, optics with a long focal length and a wide focal plane for astrometry are required. Korsch proposed a three mirror system with a long focal length and a wide focal plane in 1977. The Korsch system is one of the convincing models. However, the center of the field is totally vignetted because of the fold mirror. Therefore we consider the improved Korsch system in which the center of the field is not vignetted. Finally We obtain the diffraction limited optical design with small distortion(0.02\%).

We have experimented with the measurement of centers of star images on a CCD for investigating the accuracy of finding the positions of stars, using the algorithm for estimating the positions of stars from the photon weighted means of stars. Then we obtain the results from the experiment that the accuracy of estimation of distance between two stars has a variance of about $1 / 300$ pixel; that is, the error for one measurement is about $1 / 300$ pixel, which is almost an ideal result given by Poisson noise of photons. We also investigate the accuracy of estimation of positions with a different size of PSF. In this case also, we find that the accuracy of estimation has a variance of about $1 / 300$ pixel.

In our experiment, the separation of two stars is measured by using the image of the field on the CCD array. Precisely speaking, the lens system distorts an image of a star field, and then the estimated distance of stars includes the error by the distortion. Therefore we must correct the distortion in order to estimate the real separations of stars. Development of the algorithm which corrects the distortion of the image by the lens system is needed. These are the future work of this experiment.

\section{References}

Triebes, K., Gilliam, L., Harris, F., Hilby, T., Horner, S., Monet, D., Perkins, P., and Vassar, R. 1999, Bull. AAS, Vol. 31, p.1505

Triebes, K. J., Gilliam, L., Hilby, T., Horner, S. D., Perkins, P., Vassar, R. H., Harris, F. H., \& Monet, D. G. 2000, in UV, Optical, and IR Space Telescopes and Instruments, James B. Breckinridge and Peter Jakobsen, eds., Proc. SPIE, Vol. 4013, p. 482-492.

Yano, T., Gouda, N., Kobayashi, Y., Tsujimoto, T., Nakajima, T., Hanada, H., Kan-ya, Y., Yamada, Y., Araki, H., Tazawa, S., Tsuruta, S., Kawano, N. 2004, PASP, 116, 667-673. 